\title{
The 2014 Interferometric Imaging Beauty Contest
}

\author{
John D. Monnier ${ }^{a}$, Jean-Philippe Berger ${ }^{b}$, Jean-Baptiste le Bouquin ${ }^{i}$, Peter Tuthill ${ }^{c}$, Markus \\ Wittkowski $^{b}$, Rebekka Grellmann ${ }^{b}$, Andre Müller $^{b}$, Sridhar Renganswany ${ }^{b}$, \\ Christian Hummel ${ }^{b}$, \\ Karl-Heinz Hofmann ${ }^{d}$, Dieter Schertl $^{d}$, Gerd Weigelt ${ }^{d}$, \\ John Young ${ }^{e}$, David Buscher ${ }^{e}$, \\ Joel Sanchez-Bermudez ${ }^{f}$, Antxon Alberdi ${ }^{f}$, Rainer Schoedel $^{f}$, \\ Rainer Köhler ${ }^{g}$, \\ Ferreol Soulez ${ }^{h}$, Eric Thiébaut ${ }^{h}$, \\ Jacques Kluska ${ }^{i}$, Fabien Malbet ${ }^{i}$ \\ Gilles Duvert ${ }^{i}$, Stefan Kraus ${ }^{j}$, \\ Brian Kloppenborg ${ }^{k}$, Fabien Baron ${ }^{k}$, \\ Willem-Jan de $\mathrm{Wit}^{b}$, Thomas Rivinius ${ }^{b}$, Antoine Merand ${ }^{b}$ \\ ${ }^{a}$ University of Michigan, Ann Arbor, MI USA; \\ ${ }^{b}$ European Southern Observatory, Germany; \\ ${ }^{c}$ University of Sydney, Australia; \\ ${ }^{d}$ Max Planck Institute for Radio-Astronomie (Bonn), Germany; \\ eUniversity of Cambridge, UK; \\ Instituto de Astrofisica de Andalucia (IAA-CSIC), Spain; \\ ${ }^{g}$ Max-Planck-Institute for Astronomy (Heidelberg), Germany; \\ ${ }^{h}$ Centre de Recherche Astrophysique de Lyon, France; \\ ${ }^{i}$ Institut de Planétologie et d'Astrophysique de Grenoble, France; \\ ${ }^{j}$ University of Exeter, Exeter, UK; \\ ${ }^{k}$ Georgia State Univiersity, USA
}

\begin{abstract}
Here we present the results of the 6th biennial optical interferometry imaging beauty contest. Taking advantage of a unique opportunity, the red supergiant VY CMa and the Mira variable R Car were observed in the astronomical H-band with three 4-telescope configurations of the VLTI-AT array using the PIONIER instrument. The community was invited to participate in the subsequent image reconstruction and interpretation phases of the project. Ten groups submitted entries to the beauty contest, and we found reasonable consistency between images obtained from independent workers using quite different algorithms. We also found that significant differences existed between the submitted images, much greater than in past beauty contests that were all based on simulated data. A novel "crowd-sourcing" method allowed consensus median images to be constructed, filtering likely artifacts and retaining "real features." We definitively detect strong spots on the surfaces of both stars as well as distinct circumstellar shells of emission (likely water/CO) around R Car. In a close contest, Joel Sanchez (IAA-CSIC/Spain) was named the winner of the 2014 interferometric imaging beauty contest. This process has shown that "new comers" can use publicly-available imaging software to interpret VLTI/PIONIER imaging data, as long as sufficient observations are taken to have complete uv coverage - a luxury that is often missing. We urge proposers to request adequate observing nights to collect sufficient data for imaging and for time allocation committees to recognise the importance of uv coverage for reliable interpretation of interferometric data. We
\end{abstract}

Further author information: (Send correspondence to J.D.M. or J.P.B.)

J.D.M.: E-mail: monnier@umich.edu

J.-P.B.: E-mail: jpberger@eso.org

Optical and Infrared Interferometry IV, edited by Jayadev K. Rajagopal, Michelle J. Creech-Eakman,

Fabien Malbet, Proc. of SPIE Vol. 9146, 91461Q • ( ) 2014 SPIE • CCC code: 0277-786X/14/\$18

doi: $10.1117 / 12.2057312$

Proc. of SPIE Vol. $914691461 \mathrm{Q}-1$ 
believe that the result of the proposed broad international project will contribute to inspiring trust in the image reconstruction processes in optical interferometry.

Keywords: imaging, interferometry, aperture synthesis, VLTI, evolved stars, molecules, water, mass-loss, compressed sensing

\section{INTRODUCTION}

The vast majority of publications making use of optical interferometry data base their analysis on model fitting. This requires strong a priori knowledge on the object brightness distribution. Such a strategy breaks down in situations were the environment to be studied is complex. In order to rely as little as possible on model-dependent information, active optical interferometers are turning more frequently to true aperture synthesis like their radio counterparts. ${ }^{1}$ Images with unprecedented angular resolution and astrophysical added value have been produced and have been a source of motivation for the development of the technique (see e.g Ref. 2 or Ref. 3 ).

The Very Large Telescope Interferometer (VLTI) offers a rich combination of telescope configurations using either the Unit Telescopes ( $8 \mathrm{~m}$ diameter) or the Auxiliary Telescope (1.8m diameters). The latter have the remarkable property of being relocatable which offers a unique way to fill the uv plane. Despite this advantage, the VLTI has produced less convincing images in recent years than the CHARA interferometer, whose six telescopes are fixed in position. A possible interpretation of this fact is a combination of two effects: 1) until recently the VLTI instruments offered combined only two or three telescopes (compared to four or six simultaneous telescopes at CHARA) which makes it difficult to fill the spatial frequency plane; 2) the Observing Programmes Committee (ESO's time allocation committee) seems to regularly reject programs requesting a significant number of nights for imaging on the grounds that the added value of imaging is low. This analysis motivated the prospect to demonstrate the VLTI imaging capability using its latest instrument, PIONIER, ${ }^{4}$ which is capable of combining four telescopes, allowing very good uv coverage with modest expenditure of observing time.

The ESO Director for Science was consulted and the Director of Paranal Observatory was asked to give his authorization to use recently freed technical time to carry-out an ambitious aperture synthesis imaging observing sequence. Since there were no programs in the service mode queues nor visitors on the mountain, a slot of several nights on the three different configurations were allocated.

A few tens of community members were solicited to provide suggestions on possible targets with the following requirements: The target had to be 1) well resolved at the VLTI angular resolutions; 2) sufficiently well positioned in the sky to provide a proper uv coverage; 3) sufficiently bright to provide high signal-to-noise ratio data. Two objects were selected: the red supergiant VY CMa and the Mira star R Car. It was agreed with ESO's Paranal Director that the data would be made public to all members of the community once the study was over.

The observations were carried by Paranal observatory astronomers and telescope operators on-duty. The raw data was reduced by J.-B. Le Bouquin using the pndrs data reduction package, ${ }^{4}$ resulting in calibrated files in the optical interferometry data exchange format OI-FITS. ${ }^{5}$ Later a call for interested parties with no restriction of home institution was issued in order to gather in the same team specialists of image reconstruction and astronomers with expertise on the observed objects. Finally recognising that the image reconstruction effort would probably involve the same community teams than the ones contributing to the 2014 interferometric imaging beauty contest J. Monnier (hereafter JDM) and J.-P. Berger (hereafter JPB) decided to use the data in the beauty contest. In the following we describe the results of the image reconstruction process and the analysis of the submissions by the different parties.

\section{DESCRIPTION OF OBJECTS AND THE VLTI/PIONIER DATASET}

\subsection{General}

Observations of VY CMa and R Car were carried with the VLTI/PIONIER instrument between January 22nd and February 2nd 2014 using all possible Auxiliary telescope quadruplets (A1-J3-K0-G1, H0-I1-D0-G1, A1-C1D0-B2). PIONIER recorded data in 3 spectral bands simultaneously with central wavelengths approximately $1.61 \mu \mathrm{m}, 1.67 \mu \mathrm{m}$, and $1.73 \mu \mathrm{m}$, with $\Delta \lambda \sim 0.07 \mu \mathrm{m}(\mathrm{R} \sim 24)$. All science measurements were interleaved with calibrators measurements as close as possible in the sky in order to avoid differential birefringence-induced 

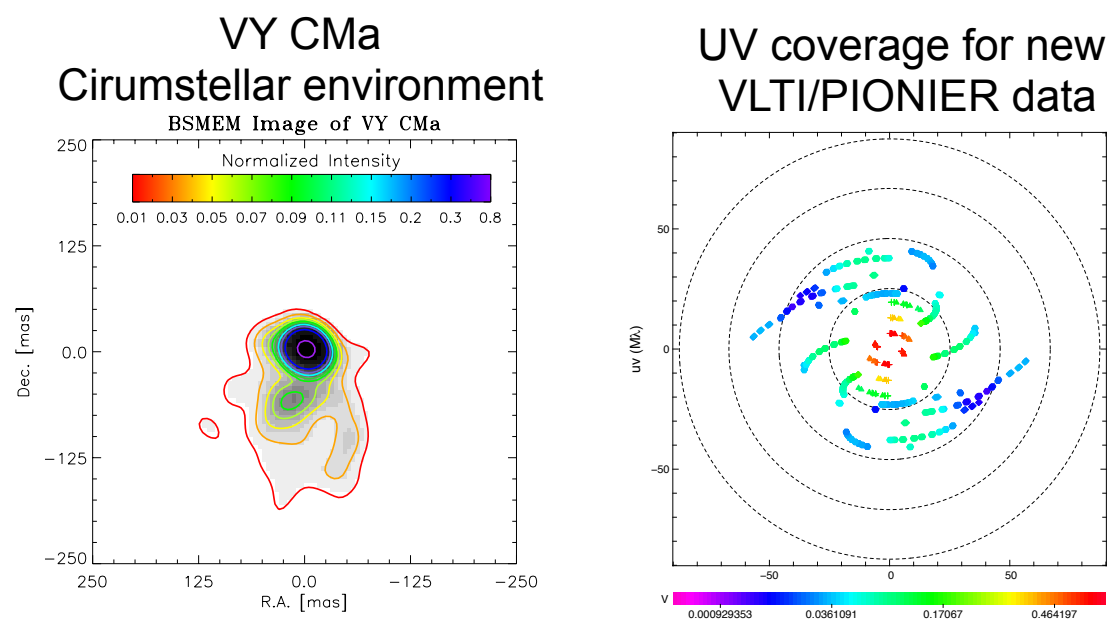

Figure 1. (left panel) This shows an aperture masking image of VY CMa taken about 6 years ago ${ }^{7}$ in order to understand the complex environment around this star. (right panel) Here we see the new uv coverage of the VLTI/PIONIER H-band data used for the Beauty contest. Note that these data probe the target on scales of $<50$ milliarcseconds, essentially allowing us to "zoom in" on the central source seen in the left panel.

biases. About 1000 visibility data points were recorded on $\mathrm{R}$ Car and 300 on VY CMa. Given the high signal to noise the average accuracy on the squared visibilities was $\approx 2 \%$ and $\approx 1^{\circ}$ on the closure phase.

Using the oft-cited definition of interferometric angular resolution of $\Theta=\frac{1}{2} \frac{\lambda}{B_{\max }}$, the VY CMa dataset possesses an angular resolution of $\Theta \sim 1.7$ milliarcseconds while the $\mathrm{R}$ Car dataset (suffering from less baseline projection) will have $\Theta \sim 1.3$ milliarcseconds resolution. This level of resolution should allow about 5-7 pixels across the photosphere of each star (each $\sim 10$ milliarcseconds diameter).

\subsection{VY CMa}

VY CMa is a famous mass-losing red supergiant (M2-5) with a thick dust shell which has been extensively imaged by ground-based adaptive optics, ${ }^{6}$ aperture masking, ${ }^{6,7}$ and the Hubble Space Telescope. ${ }^{8}$ The star itself has been scrutinized using 2 - and 3 -telescope data from long-baseline optical interferometers, ${ }^{9,10}$ with a VLTI/AMBER near-continuum diameter measurement of 11.3 mas (Ref. 10). This star has a complex environment and is a perfect target for this unique VLTI/PIONIER imaging project.

Figure 1 contains an archival aperture masking image of the circumstellar environment (OIFITS provided by P. Tuthill, see also Ref. 7) and the uv coverage of the new VLTI/PIONIER dataset is also shown. Figure 2 show a summary of the visibilities and closure phases measured for VY CMa using 3 different configurations of the ATs. Full observing details will be forthcoming in a future paper.

\subsection{R Car}

$\mathrm{R}$ Car is a bright Mira variable with spectral type M5-M8. Strong water (and possibly CO) bands flank the Hband atmospheric window centered on $1.65 \mu \mathrm{m}$. While no infrared diameter measurements have been previously reported, R Car was measured in the red (700-900nm) using aperture masking ${ }^{11}$ combined with spectroscopy, and these authors found a characteristic (gaussian FWHM ) size ranging from 15-20 milliarcseconds depending on the spectral channel, with variations likely due to the differing strengths of molecular layers surrounding the star. Recent work with VLTI/AMBER ${ }^{12}$ confirmed earlier suggestions ${ }^{13}$ that Mira variables are surrounded by complex layers of molecular emission of especially water and CO.

Figure 3 contains a near-IR spectrum of a star with the same spectral type as R Car (no actual spectrum could be located) along with the uv coverage of the new VLTI/PIONIER dataset. Figure 4 show a summary of 


\section{VY CMa VLTI/PIONIER Dataset}
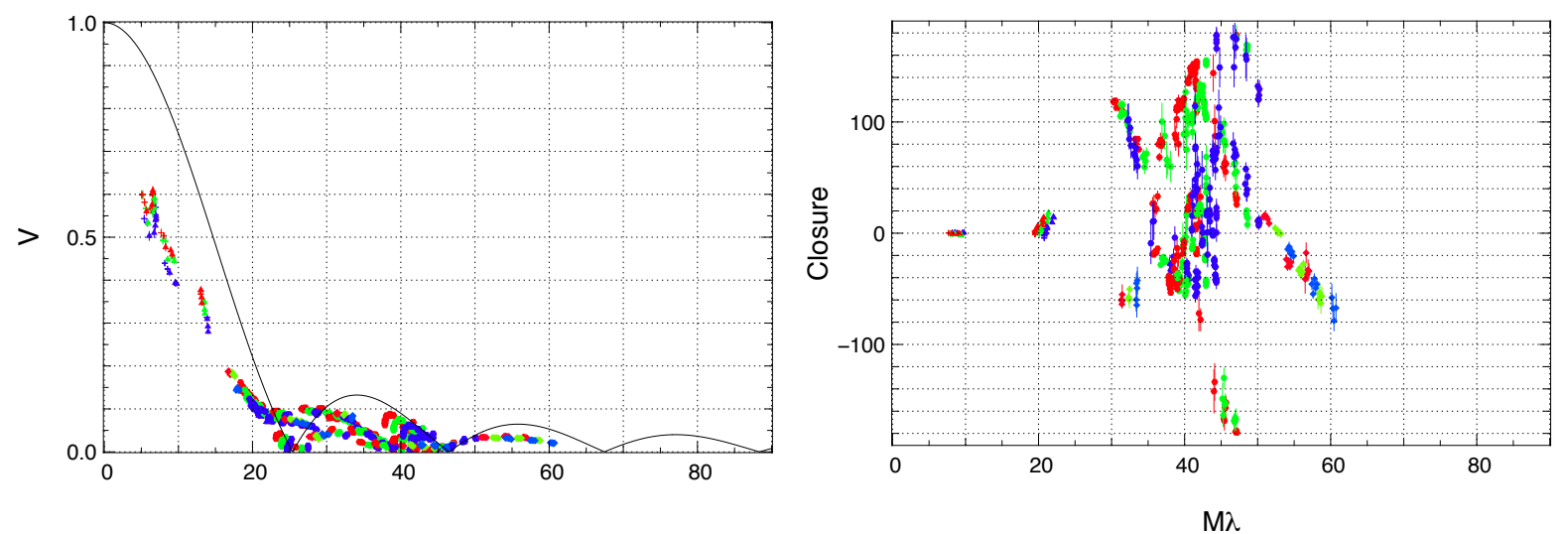

Figure 2. (left panel) The VLTI/PIONIER visibility data for VY CMa are shown here. (right panel) The measured closures phases are plotted as a function of length of the longest baseline in a given closing triangle. For both panels, the colors show the 3 different spectral channels.
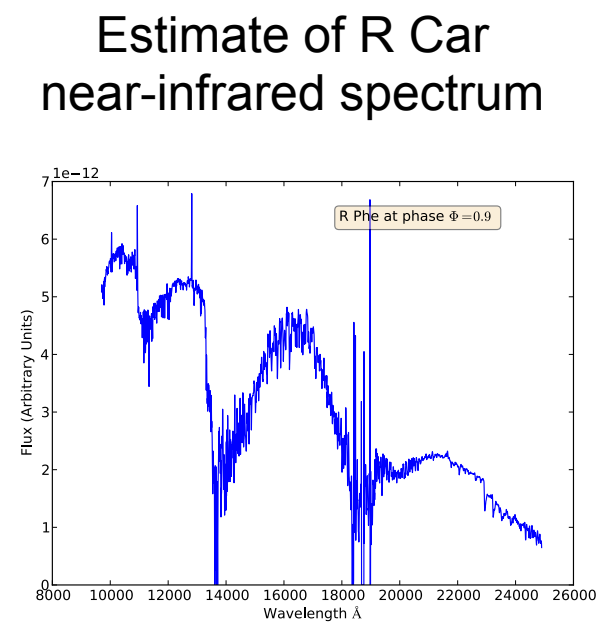

\section{UV coverage for new VLTI/PIONIER data}

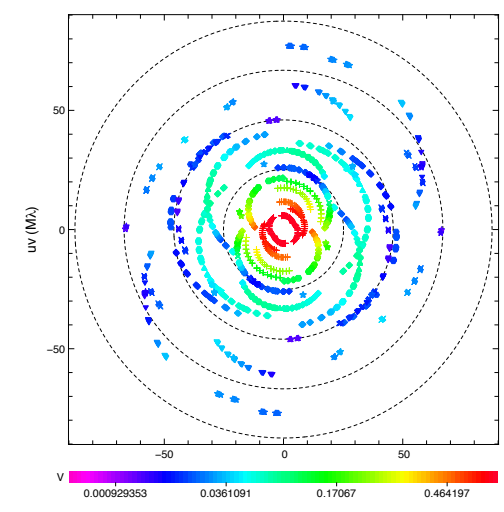

Figure 3. (left panel) This shows an estimate of the near-IR spectrum of R Car at the phase of the new VLTI/PIONIER observations, based on a different star with similar spectral type. (right panel) Here we see the new uv coverage of the VLTI/PIONIER H-band data used for the Beauty contest. This uv-coverage is dense and covers a range of scales (to probe both the compact stellar photosphere and the extended molecular envelope), offering the opportunity for an unprecedented look at a Mira Variable star.

the visibilities and closure phases measured for R Car using 3 different configurations of the ATs. Full observing details will be forthcoming in a future paper.

\section{THE ENTRIES}

\subsection{Overview}

Ten groups submitted image reconstructions for the beauty contest, with some groups consisting of only a single member while others represented a larger team effort. Table 1 contains a summary of the entries, including the 


\section{R Car VLTI/PIONIER Dataset}
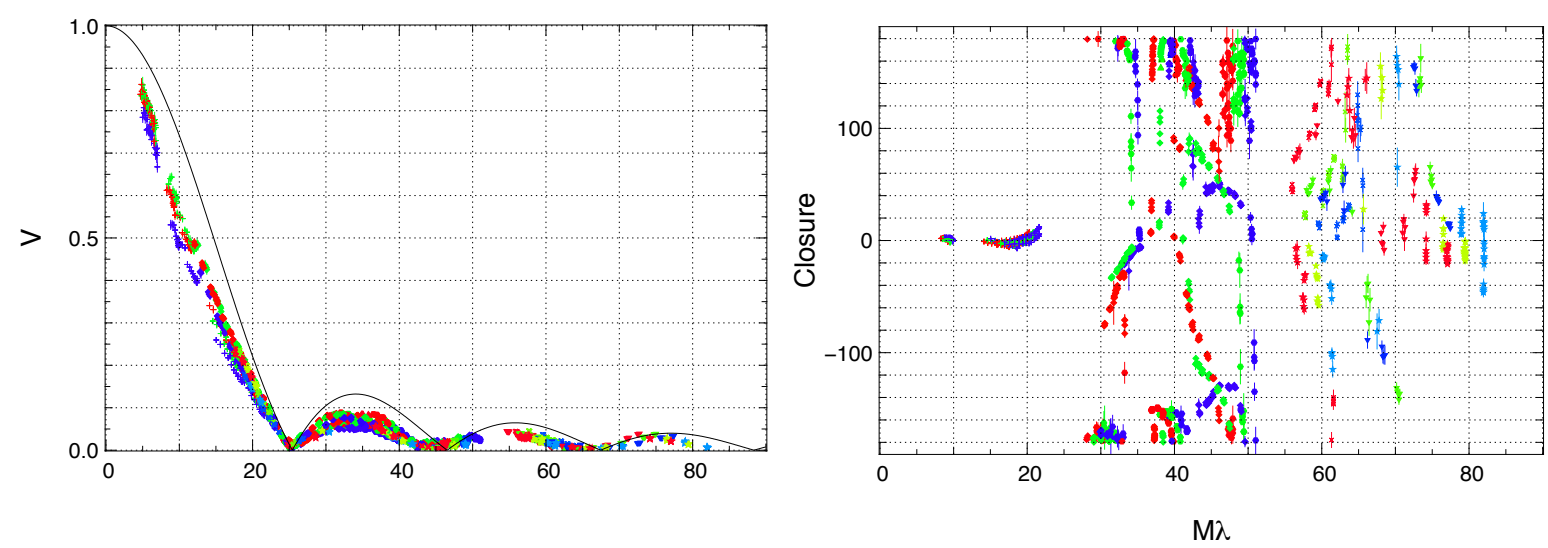

Figure 4. (left panel) The VLTI/PIONIER visibility data for R Car are shown here. (right panel) The measured closures phases are plotted as a function of length of the longest baseline in a given closing triangle. For both panels, the colors show the 3 different spectral channels.

lead investigator, the algorithm used, and some details regarding use of priors and/or wavelength regularizers. All groups were asked to provide text describing their methodology and including a short discussion of their images, including which image features were likely to be real features and which features were likely to be artifacts. We have only lightly edited these self-descriptions and thus the subsections below vary in length and detail, with some entrants providing only a paragraph of description while others providing much greater elaboration. A more consistent and complete treatment will be contained in a future refereed paper.

Table 1. Overview of Interferometric Imaging Beauty Contest Entries

\begin{tabular}{|l|l|l|l|l|l|}
\hline \multirow{2}{*}{ Submitter } & Algorithm & \multicolumn{2}{|c|}{ Prior? } & \multicolumn{2}{c|}{$\lambda$ Regularization? } \\
& & VY CMa & R Car & CMa & R Car \\
\hline Hummel & PEARL/CLEAN & No & No & Gray & Gray \\
\hline Hofmann & IRBis & No & No & No & No \\
\hline Young & BSMEM & 2 Gaussians & LDD+Gaussian & No & No \\
\hline Sanchez & BSMEM & Extended Gaussian & UD+2 Gaussians & Gray & Gray \\
\hline Köhler & MIRA & No & No & No & No \\
\hline Soulez & MIRA3D & No & No & Yes & Yes \\
\hline Kluska & MIRA-SPARCO & Gray image & Gray image & via Prior & via Prior \\
\hline Duvert & WISARD & No & No & No & No \\
\hline Kraus & SQUEEZE/MACIM & No & No & No & No \\
\hline Kloppenborg & SQUEEZE-poly $\lambda$ & No & No & Yes & Yes \\
\hline
\end{tabular}

Figure 5 show the reconstructed images of VY CMa for all 3 spectral channels by the 10 groups who participated in the contest. Likewise, Figure 6 presents the results for R Car. We only show the inner 25 milliarcsecond field-of-view here. For presentation and comparison purposes we have interpolated each image onto a common 1 milliarcsecond grid, typically 3-5 times more fine than the grid resolution of the submitted entries. The interpolation was done in the Fourier plane by introducing extra zero-padding before an inverse Fourier Transform. 
Each image here was normalized to the peak surface brightness and a color bar is provided for each figure.

\subsection{Christian Hummel using CLEAN/PEARL}

The multi-wavelength CLEAN algorithm PEARL ${ }^{14}$ was used to reconstruct images of VY CMa and R Car. However due to PIONIER's limited wavelength coverage, there was no real advantages for this method and thus the final submitted entries were gray, identical in each of the 3 narrow spectral bands. No cleaning windows were used, just about 5 phase self-calibration iterations with 100 CLEAN components added to the model in between (similar to difmap).

Unconvolved images of the CLEAN components were provided for the contest. JDM smoothed these with a gaussian of full-width-at-half-maximum (FWHM) 2.0 milli-arcseconds, a scale chosen to be roughly equal to the angular resolution of the interferometer and one that lended itself to comparison with the other maps. For VY CMa, Dr. Hummel believes the east-west extensions of VY CMa is real. In the case of R Car, the feature 10 mas east of the center was thought to likely be an artifact.

\subsection{Karl-Heinz Hofmann et al. using IRBis}

Karl-Heinz Hofmann, Dieter Schertl, and Gerd Weigelt submitted multi-wavelength reconstructions using the algorithm IRBis. ${ }^{15}$ The $\chi^{2}$ values for the visibilities and closure phases are $\sim 1$ for reconstructions for both objects in all wavelengths.

For VY CMa, these investigators found two dominant extended spots and fainter structures to be visible. These structures did not look like a sharp stellar disk. The resolved structures may be caused by dust clouds in front of the star as, for example, in the dust envelope of the carbon star IRC+10216. The intensity ratio of the two dominant extended spots seems to show some wavelength dependence. A faint nebulosity background at the $\sim 5 \%$ level is visible and is probably the innermost region of the circumstellar envelope detected by previous bispectrum speckle interferometry observations. ${ }^{16}$ Note the largest circular boundary in the background is caused by a binary mask used in the reconstruction process (see Ref.15).

For R Car, the reconstructions show an almost round and sharp stellar disk in each spectral channel, although the stellar disks have slightly different diameters in the three different spectral channels. Fully Darkened Disk (FDD) fits to the measured visibilities yield the following diameters: spectral channel $1.61525 \mu \mathrm{m}$ : 11.63 mas; spectral channel $1.66785 \mu \mathrm{m}$ : 11.50 mas; spectral channel $1.71995 \mu \mathrm{m}$ : 11.45 mas. Indeed, the reconstructed images show the same wavelength dependence of the diameter. Such diameter variations are also observed in other Miras and predicted by Mira star models. ${ }^{17}$ Several spots, near the detection limit, are visible, which are approximately identical in each spectral channel. In particular, two spots (in the middle of each disk) with a separation of 3 mas are visible; the fainter spot of this double spot seems to become weaker with respect to the brighter one with increasing wavelength. An approximately round weak background $(\sim 2-6 \%$ of peak flux $)$ outside the disk is visible with a radius of $\sim 2$ stellar radii.

\subsection{John Young et al. using BSMEM}

John Young and David Buscher submitted entries using the BSMEM algorithm. The BSMEM (BiSpectrum Maximum Entropy Method) software was first written in 1992 to demonstrate image reconstruction from optical aperture synthesis data. ${ }^{18}$ It has been extensively enhanced and tested since then, ${ }^{19}$ although there have been no changes of late. The code used for this year's contest entry is essentially identical to that employed for the 2010 and 2012 contests.

The algorithm applies a fully Bayesian approach to the inverse problem of finding the most probable image given the evidence, making use of the Maximum Entropy approach to maximize the posterior probability of an image. BSMEM is available free-of-charge to the scientific community on submission of the academic license agreement at http://www.mrao.cam.ac.uk/research/OAS/bsmem.html.

BSMEM uses a trust region method with non-linear conjugate gradient steps to minimise the sum of the $\log$ (likelihood) (chi-squared) of the data given the image and a regularization term expressed as the Gull-Skilling entropy $\sum_{k}\left[I_{k}-M_{k}-I_{k} \log \left(I_{k} / M_{k}\right)\right]$. The model image $M_{k}$ is usually chosen to be a Gaussian, a uniform 

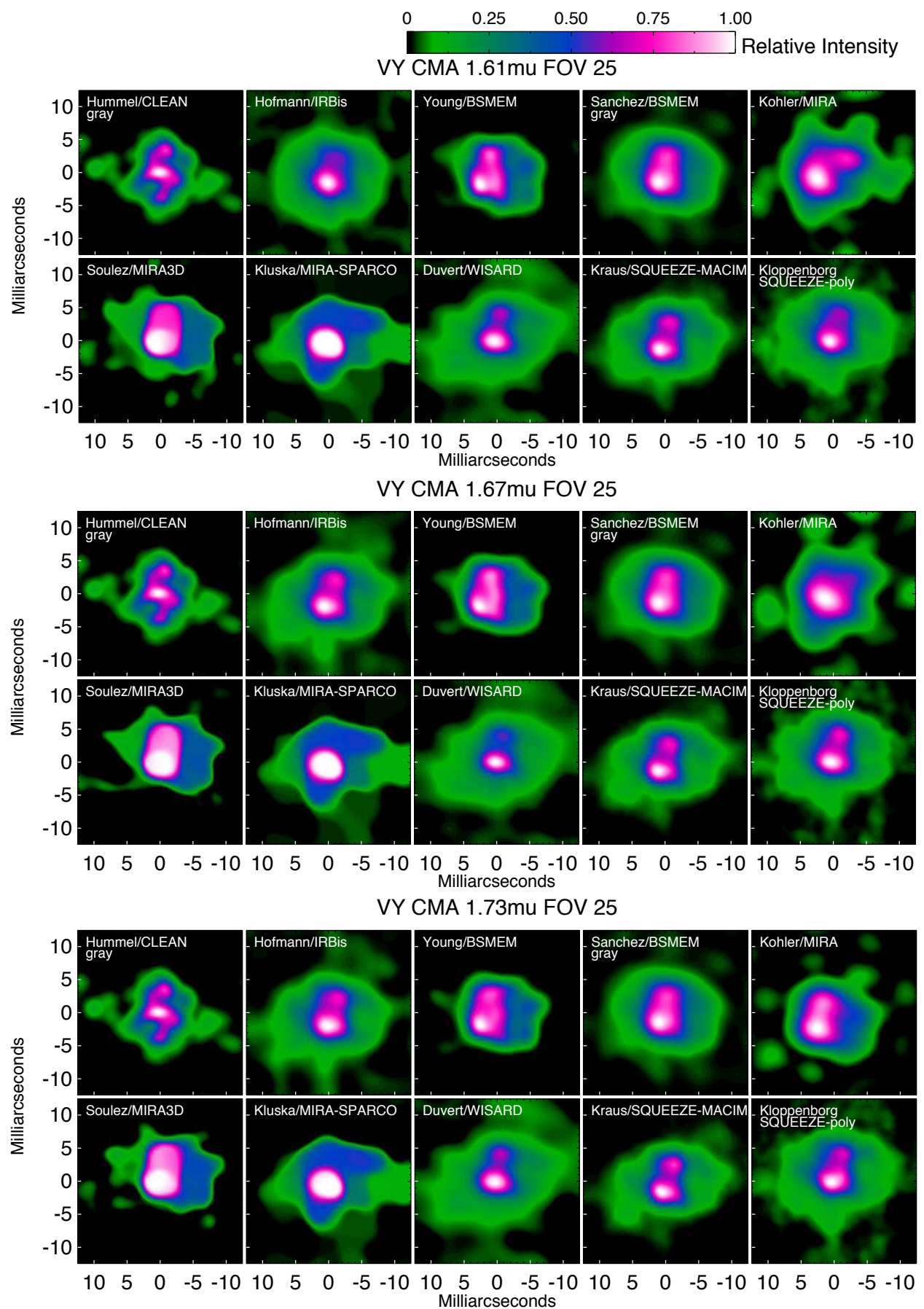

Figure 5. This panel shows all the images from the contestants for VY CMa, in each of the three narrow spectral bands. East is left, North is up and the field-of-view is $25 \times 25$ milliarcseconds. The images are scaled by the peak surface brightness. The color table is non-linear and a color bar gives a more quantitative feeling for the surfaces brightness levels for each entry. The effective angular resolution varied between the entries and no attempt was made to convolve entries to a common angular resolution (see text for more detail). 
disk, or a delta-function centered in the field of view, which conveniently fixes the location of the reconstructed object (the bispectra and powerspectra being invariant to translation). This type of starting model also acts as a support constraint by penalising the presence of flux far from the centre of the image. An important advantage of BSMEM is the automatic Bayesian estimation of the hyperparameter that controls the weighting of the entropic prior relative to the likelihood.

For both R Car and VY CMA, the supplied VLTI data were split into three wavebands, ignoring the small night-to-night wavelength variations. The aperture masking data on VY CMa were not used in generating the submitted images, owing to the apparent mismatch with the short-baseline VLTI data.

Simple Gaussian or uniform disk prior models were insufficient to allow BSMEM to converge to a solution for these datasets, so an initial model-fitting step was carried out. For R Car the model chosen was a circular Hestroffer limb-darkened disc (to represent the stellar photosphere) plus a resolved circular component to represent the circumstellar emission. For VY CMa the chosen model comprised a compact ( 7.2 mas FWHM) circular Gaussian component plus a 80-mas FWHM circular Gaussian (the smallest size that was essentially resolved out on the shortest VLTI baselines). An image was generated from the best-fitting model at each waveband. The sharp edge in the R Car model images was smoothed by convolving with a 2-pixel Gaussian.

For all six datasets, it proved very difficult to get BSMEM to converge to a solution. In the end, this was achieved for VY CMa by the unsatisfactory method of removing all data with SNR below 5 then multiplying the error bars on the remaining data by a factor of 1.3 or 1.4. This is likely to have resulted in a slight overregularization of the reconstructed images. The same approach was used for $\mathrm{R}$ Car but it was also found necessary to strengthen the support constraint by artificially reducing the flux of the Gaussian model component (e.g. at $1715-1738 \mathrm{~nm}$ from $104 \%$ down to $5 \%$ of the stellar disk flux).

The VY CMa images were obtained without using a flux normalization constraint, as significant emission is expected from this extended object to fall outside the interferometric field defined by the spectral bandpass, but within the optical field of view of the beam combiner. The fluxes in the submitted VY CMa images are 0.71, 0.69 , and 0.68 for the $1610-1625,1663-1678$ and $1715-1730 \mathrm{~nm}$ wavebands respectively.

For VY CMa, these investigators believe the following features are real: 1) the brightness difference between the East and West halves of the central component; 2) the compact bright feature at the SE corner of the central component; and 3) the faint extension to the East extending from the NE corner of the central component.

For R Car, these investigators judged that the following features in the submitted images are real: 1) the near-circularity of the stellar disk; 2) the extent and relative flux of the circumstellar shell; 3) the brightest 3 compact features on the stellar surface at 1610-1633 nm and 1663-1685 nm, and the brightest single feature at 1715-1738 nm. The circumstellar emission may well be somewhat clumpy, but this group does not believe that any of the individual clumps visible in the reconstructed images represent reality.

\subsection{Joel Sanchez-Bermudez et al using BSMEM}

Joel Sanchez-Bermudez, Antxon Alberdi, and Rainer Schoedel also submitted entries using the BSMEM software. These users have participated in this contest for the first time. They are new comers in the optical interferometry community, with some publications in the last two years including reconstructed NIR interferometric images making use of BSMEM and MACIM. The final reconstructions included all the channels presented in each data set analyzed (grey). For each star, initial models (in the form of *.fits files) were created before beginning the maps reconstruction with BSMEM. Those prior models were created via a direct model fitting to the squared visibilities $\left(\mathrm{V}^{2}\right)$ and closure phases $(\mathrm{CPs})$. The procedure used was the following:

a. The original OI-FITS ${ }^{5}$ files were split into the different epochs that they contained.

b. Epochs with similar sampled spatial frequencies were merged into new * .oifits files. This step allowed us to independently analyze the extended and compact components of the source.

c. For each of the new OI-FITS files, geometrical models were fitted to the interferometric observables. Each model was reconstructed starting with a single component (e.g. Gaussians, disks, etc.), and we continued adding components to reduce the difference between data and models (via a $\chi^{2}$ minimization). 
d. Having reached a relatively good approximation to the data, an image was created with the best-fit model.

e. The best-fit models were then loaded to BSMEM as prior images from which the image reconstruction process started.

The aforementioned model fitting allowed valuable information to be inferred relating to the expected morphology of the sources. The model fitting for the extensive PIONIER datasets was complicated since the trends of the CPs suggests the existence of many different compact components. For example, for the R Car data, the above-described procedure distinguished at least three components: an extended disk (the source diameter) and two compact features represented by Gaussians. The pixel size for the image reconstruction was selected to fulfill the Nyquist criterion. The field of view was selected to be big enough to include all the sampled spatial frequencies. All the reconstructed images were convolved with a beam with a FWHM similar to the angular resolution of the interferometer used. After the reconstruction process in BSMEM, a reduced $\chi^{2}$ of the best-fit images of around 20 and 6 was obtained for the PIONIER VY CMa and the PIONIER R Car data, respectively. The SNR obtained for all the reconstructed images was around 100. Hence, all the features below $1 \%$ of the flux peak can be considered artifacts.

\subsection{Rainer Köhler using MIRA}

Rainer Köhler wanted to participate in the Imaging Beauty contest as a way to find out whether an inexperienced user would be able to reconstruct images that were comparable to those produced by experts. He used the software package MIRA $^{20}$ written by Eric Thiébaut and his group.

For the image reconstruction, an initial image was used consisting of a uniform disk with a diameter of 8 mas. Two regularization terms were tried: quadratic smoothness and total variation. The image obtained with quadratic smoothness looks more realistic, and these are submitted here.

It was difficult to judge which features in the images are real. This investigator was confident that the bright peak near the center of $\mathrm{R}$ Car is real. The rim might be brighter at longer wavelengths (i.e. redder), but the investigator was not sure about this. With VY CMa, the two large bright spots on the east side of the disk was judged real, but it might also be one even larger spot. In the shortest wavelength channel, there is also a spot on the east side of the disk, which may be real. For both stars, there was no claim that any of the faint features outside of the stellar disks are real detections.

\subsection{Ferreol Soulez et al. using MIRA3D}

R Car and VY CMa datasets have been reconstructed using MiRA3D ${ }^{21}$ by Ferreol Soulez and Eric Thiébaut. As no information were provided about the bandwidth of each spectral channel, for each spectral channel a gray level image was reconstructed centered on its nominal wavelength. Both datasets were reconstructed using a transpectral total variation regularization that favors 1 ) object with sharp edges as $2 \mathrm{D}$ total variation and 2) that these edges are located at a similar position in each spatial channel.

The VY CMa object is enshrouded in a large nebula much wider than the interferometric field of view that introduce a large amount of incoherent flux. In this reconstruction this incoherent flux was considered to represent $25 \%$ of the total flux. Hence the reconstructed image accounts only for the $75 \%$ coherent flux and is then normalized to a total flux of 0.75 in each channel. This very coarse estimation of the incoherent flux may produce low flux patches scattered in the field of view. These artifacts prevents the interpretation of the environment around the star.

This star is a more or less elliptical disk (about 12.5 mas in diameter). This disk is not homogeneous: the east part appears to be brighter and a very bright spot is located on the south east part. This resolved spot has a width of 4 mas. In addition, as indicated by its spectrum, this bright spot is also much hotter than the rest of the star.

The R Car object is composed of a disk of about 10 mas in diameter. This disk is not uniform and especially there is a marginally resolved brighter spot ( $14 \%$ brighter) of about 3 mas in width located 1 mas north of the center of the disk. This disk is surrounded by an approximately circular shell of about 22 mas in diameter. The optical thickness of this shell differs from one spectral channel to another, especially it seems to be more optically thin in the $1.67 \mathrm{~m}$ than in the $1.61 \mu \mathrm{m}$. 

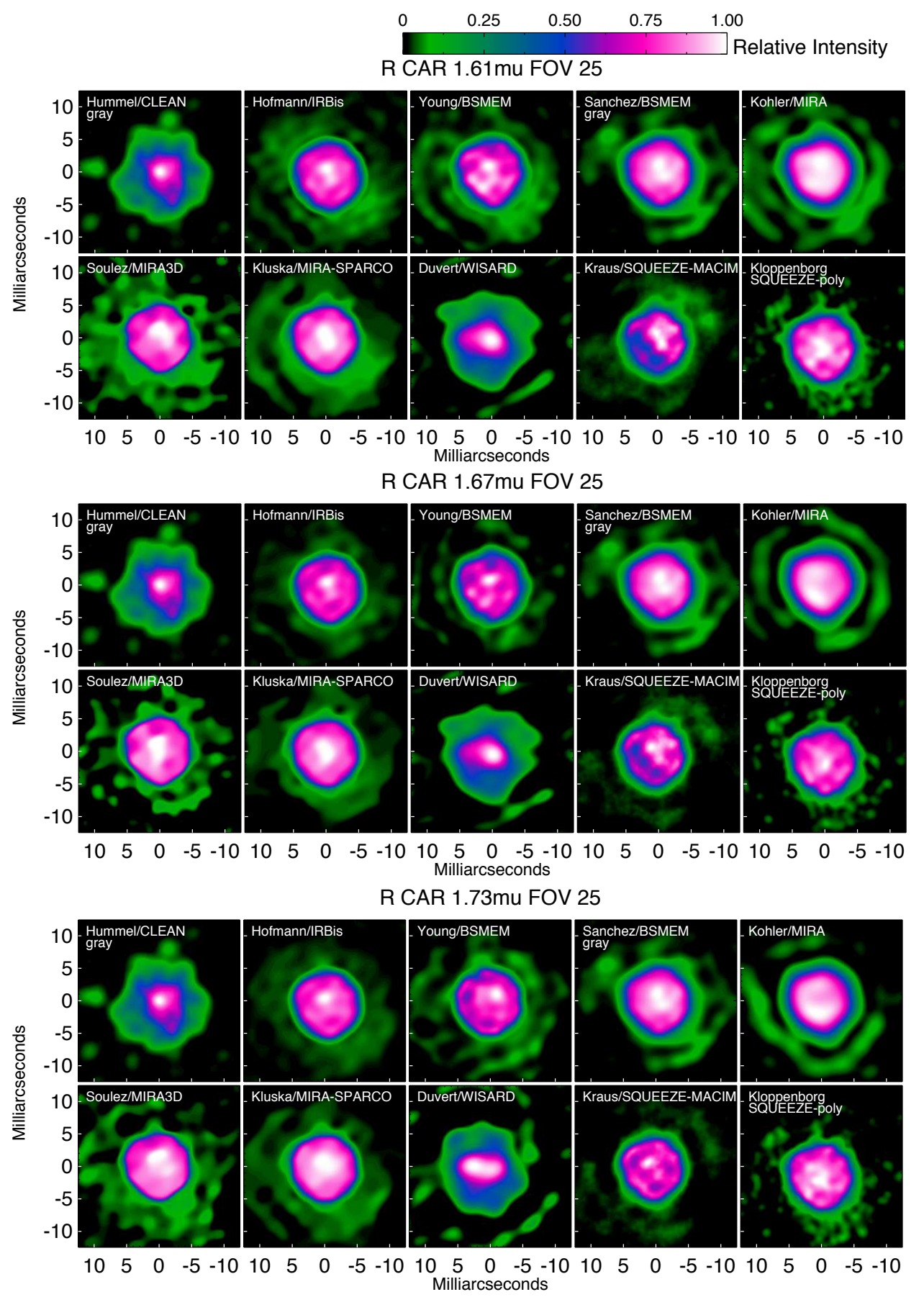

Figure 6. This panel shows all the images from the contestants for R Car, in each of the three narrow spectral bands. East is left, North is up and the field-of-view is $25 \times 25$ milliarcseconds. The images are scaled by the peak surface brightness. The color table is non-linear and a color bar gives a more quantitative feeling for the surfaces brightness levels for each entry. The effective angular resolution varied between the entries and no attempt was made to convolve entries to a common angular resolution (see text for more detail). 


\subsection{Jacques Kluska et al using MIRA-SPARCO}

Jacques Kluska and Fabien Malbet decided to make the reconstructions using the SPARCO method ${ }^{22}$ with MiRA. ${ }^{20}$ A uniform disk model was used for the central star for both the objects. A two-step approach was chosen to obtain the final images. First, a parametric model of a UD (Uniform Disk) was subtracted from the image and the image reconstruction was allowed to converge. Second, the computed image of the UD was added back to the previously reconstructed image. This resulting image was set as a prior to let the algorithm converge in a normal case (without a parametric model). The reconstructions are first made with all the channels together. Then, each channel is reconstructed separately starting with the same prior which is the image reconstructed for all the channels.

Here, more details will be provided on the parametric fitting process. First, a model was fitted with LitPro of a UD, a Gaussian and a background on both the objects. The results of this model fitting indicates the size of the UD and its flux per channel. This is used for subtracting the star from the image, with a separate fit and subtraction in each spectral channel. Channel 1 have the wavelengths around $1.61 \mu \mathrm{m}$, channel 2 around $1.66 \mu \mathrm{m}$ and channel 3 around $1.71 \mu \mathrm{m}$ - the channel bandwidth is $0.05 \mu \mathrm{m}$. For VY CMa, the following fits were found: a UD size of 12.6mas for channel 1 (13.5mas and 14.4mas for channel 2 and 3) and a flux ratio of about $44.9 \%$, $48.6 \%, 50.0 \%$ for channel 1, 2 and 3 (respectively). For R Car, a mean UD size of 10.3mas (weak variations) was found with a flux ratio about $40.8 \%, 47.5 \%$ and $50.9 \%$ (for channel 1, 2 and 3 respectively).

The regularizations used are Total Variation and Smoothness. In order to determine the right hyper parameter value $\mu, \mathrm{L}$ curves were made. A value of $\mu=1600$ was found for Total Variation and $\mu=1.3 \times 10^{9}$ for Smoothness (see Fig. 7).

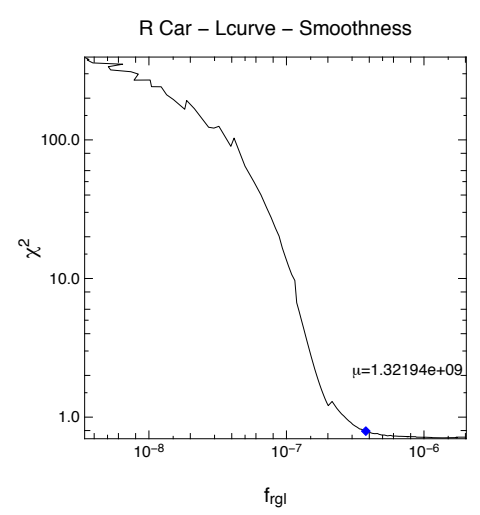

Figure 7. Example of L curve for R Car for the smoothness regularization.

For R Car, the flux ratios found from the parametric fits were used to make one image per spectral channel starting with flux in the central pixel only. A total image was computed adding the obtained image with the image of the UD. The algorithm converged using total variation, conserving big gradients made by the UD if there is no need to change them. The spots inside the UD are of the size of the beam and can be considered as really present. There is also a halo surrounding the UD that is changing in total flux with respect to spectral channel. Its shape is consistent between the channels even if there are changes for the channel 2.

For VY CMa, a total variation regularization with a hyperparameter $\mu$ value of 1600 was used. Using the same method as for $\mathrm{R}$ Car, an image was reconstructed with adding an UD of 12.6mas. Then an image reconstruction was started with the sum of the reconstructed image and the UD image (weighted by the fluxes fitted by LitPro). The shapes are very different from those reconstructed for R Car, with one bright central spot surrounded by an asymmetric envelope. The flux ratio between the spot and the envelope is changing with wavelength. This was indicated by the parametric fit done before the image reconstruction process (envelope-to-total flux ratio and size changing with the channel).

These investigators noted that a range of image details could be obtained by changing the strength and nature of the regularizer, but further elaboration is beyond the scope of this first look. 


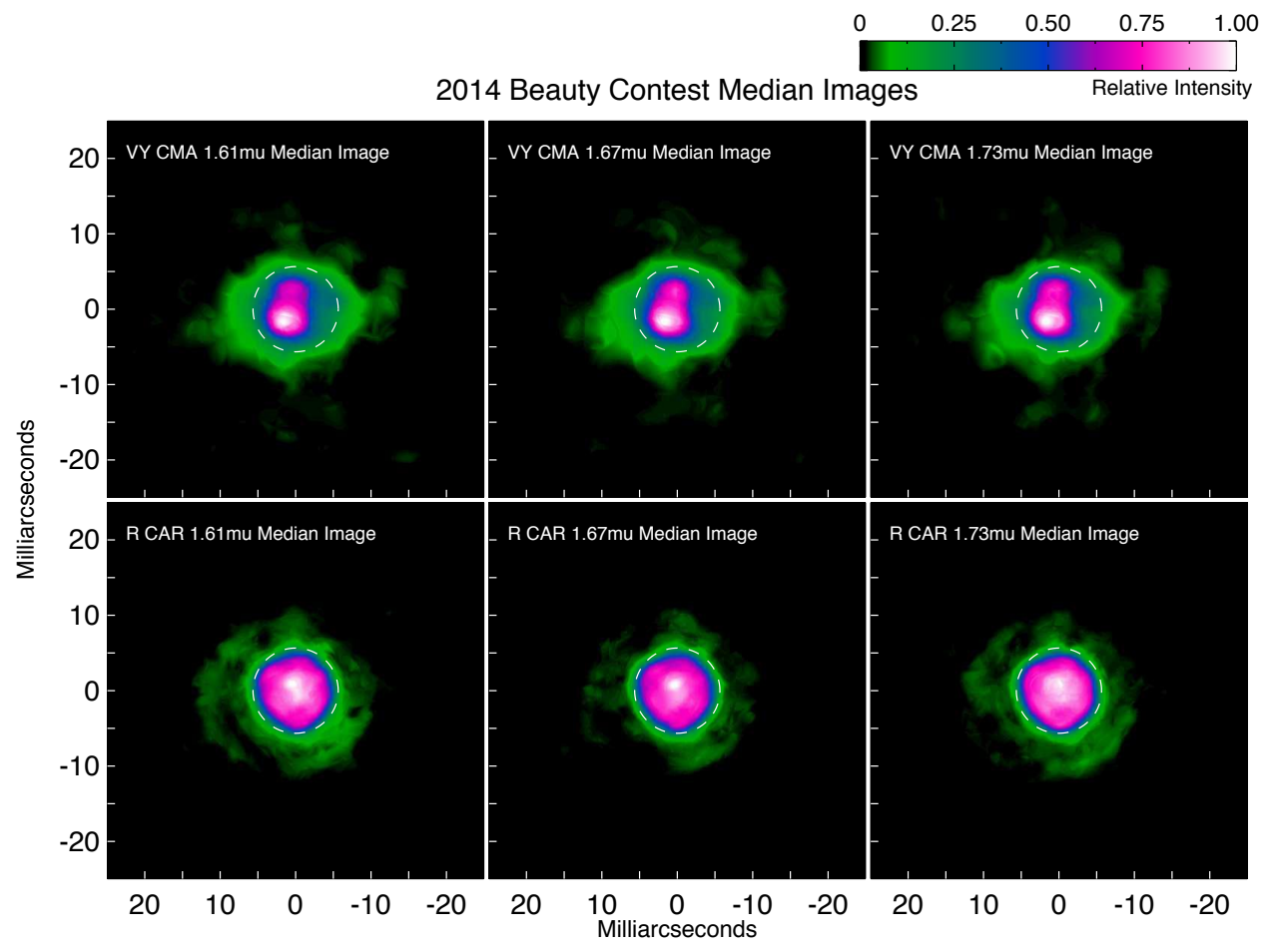

Figure 8. Following the procedure outlined in text, we have constructed a "median" image for each star at each wavelength channel. From the features in these images, we hope to correctly discriminate between "real" image features and reconstruction artifacts.

\subsection{Gilles Duvert using WISARD}

The images of R Car and VY CMa have been reconstructed with WISARD, ${ }^{23,24}$ as provided on the JMMC webpages (http://www.mariotti.fr/wisard_page.htm). The algorithm is currently written in IDL (GDL, the free IDL language interpreter, is known to handle WISARD correctly) and has been slightly modified since its first presentation, ${ }^{25}$ mainly to facilitate the reading of OI-FITS files, ${ }^{5}$ and to better support the n-telescope case.

WISARD can be seen as a two-step image reconstruction program. The last step provides a regularized reconstruction where one minimizes a two-term chi-square expression, one term being the distance to the dataset (likelihood term) and the other a distance to "some a-priori knowledge" (the prior). The many possible priors include the positivity of the reconstructed image, its smoothness, or the preservation of unresolved point sources.

The unique feature of WISARD (at least in the point of view of GD) resides in its first step. Indeed, WISARD uses the simultaneously obtained square visibility and closures to 'guess' an intermediate data set of "myopic" complex visibilities, because this transformation will always leave some phases unknown. However, as the number of telescope increases, the percentage of unknown phases diminishes rapidly, from $66 \%$ with a 3 -telescope experiment, to $30 \%$ for 6 telescopes. The reconstruction procedure then reconstructs both an image of the object (using the priors on the object) and a set of perturbation phases (the unknown phases being the sum of the phase of the reconstructed object plus these perturbation phases).

Observational datasets are rarely made of complete sets (in the WISARD sense, i.e., having all the $\mathrm{V}^{2}$ and all the closures simultaneously for $\mathrm{N}$ telescopes). Thus, only a part of the information in the PIONIER datasets was useable by WISARD. Similarly, we did not use the additional aperture masking data provided by P. Tuthill for lack of information about the correspondence of baselines in $\mathrm{V}^{2}$ and closures in their OI-FITS data.

WISARD being at the moment a monochromatic image reconstruction program only, each of the 3 channels available in the PIONIER data of this Beauty Contest was imaged separately. Both objects were reconstructed with the default procedure available in WISARD, no guess image, spike-perserving regularization (/WHITE 


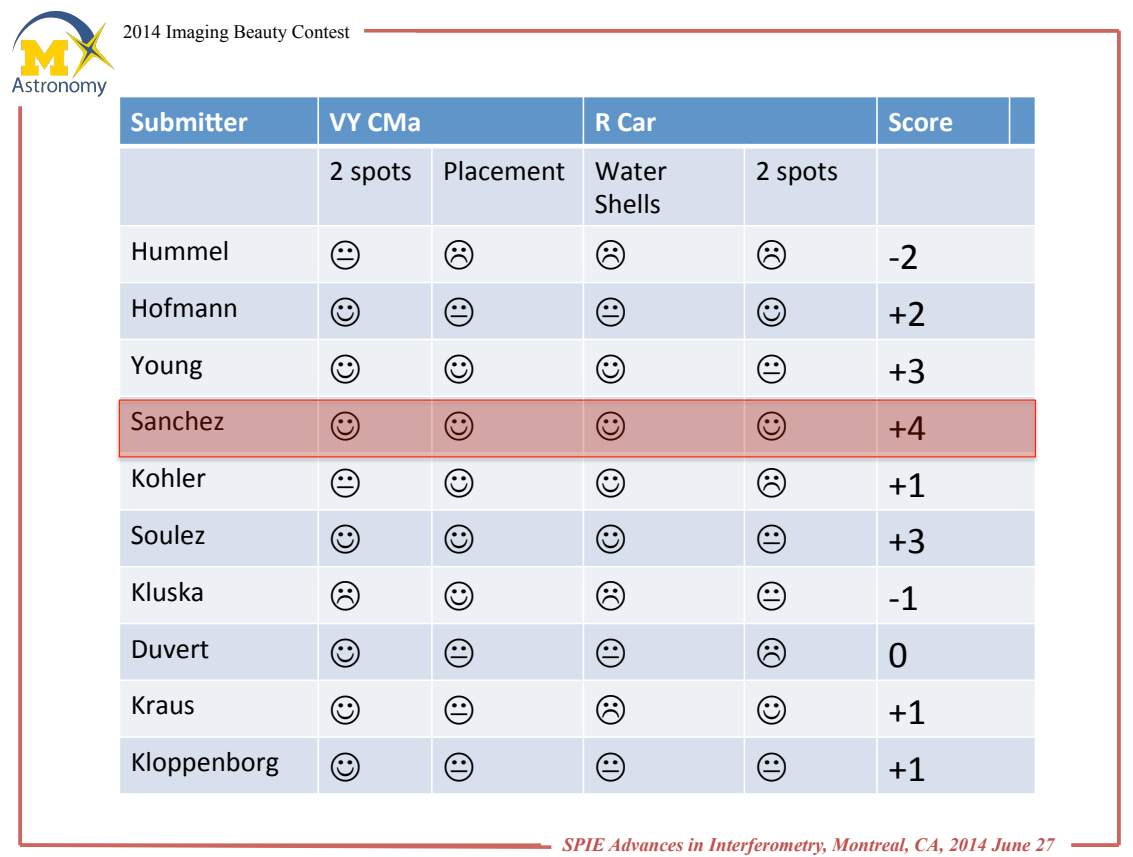

Figure 9. Snapshot of the powerpoint slide containing the judgment table presented by JDM/JPB at the 2014 SPIE meeting in Montreal.

option), 30 mas field of view for R Car (40 mas for VY CMa), $128 \times 128$ pixels in the FOV, and 1000 iterations. The final $\chi^{2}$ was $\sim 2$ for R Car and $\sim 3-4$ for VY CMa, the latter possibly due to visibility biases arising from the fully-resolved nebulous emission around this star. Channels 1 and 3 give systematically worse $\chi^{2}$ than the (central) channel 2. Trying to reconstruct images with a gray model, on all the channels at once, give much worse $\chi^{2}$ for R Car, showing that the shape of the object indeed change with wavelength. The differences are much less pronounced for VY CMa.

The reconstruction for $\mathrm{R}$ Car shows a roundish shape of approximently the size $(\sim 12$ mas $)$ one could fit as a linear limb-darkened disk in the data. One sees a central bright spot (elongated E-W) and a rapidly decreasing flux towards the outer radius of the star, like a very strong (latitude-dependent?) limb-darkening. Hints of further limb-brightening on an outer shell are visible on the composite tricolor image, at a radius of $\sim 5.9$ mas. It is not straightforward to determine which image features are "real". The reconstruction is by nature very sensitive to the precision in the phases and the underlying closures from which they are estimated. Data may be biased. Inadequate priors can be used. Only by comparing reconstructions from different algorithms, such as in this beauty contest - in other terms, only in providing some kind of "biodiversity" — can one make his/hers mind about the true nature of the object observed.

It has already been said for VY CMa that some large emission was present which was not recoverable by WISARD. The reconstruction shows distinctly two emission sources, with a "secondary" at P.A. -10 (alternatively, an E-W absorption lane in front of a stellar surface), plus an oval emission area with a distinct "plume" in the NW. Interestingly, this feature, present on all channels, is at a smaller scale similar to "the curved, nebulous morphology of the dust plume extending to the northwest" in Monnier et al. (Ref. 6; see their Figure 4). One may wonder if this interpretation made of the large-scale feature holds at the spatial scale imaged here. Again, astrophysical modelling should be envisioned only if a majority of image-reconstruction programs confirm these features. 


\subsection{Stefan Kraus using SQUEEZE/MACIM}

Stefan Kraus submitted image reconstructions using the SQUEEZE ${ }^{26}$ software package, which uses the Monte Carlo image reconstruction engine that was introduced by MACIM ("MArkov Chain Imager"; Ref. 27).

The visibility profiles of R Car and VYCMa show clear evidence for the presence of an extended emission component. Therefore, the images were reconstructed with a large field-of-view (FOV) of 80 mas, which allows the image reconstruction algorithm to place flux elements also on relatively large spatial scales. Given that this FOV is still smaller than the fiber entrance of PIONIER with the ATs ( $~ 300$ mas), the image reconstruction process was allowed to also have the presence of an overresolved flux component. The fractional contribution of this uncorrelated flux to the total flux $\left(f_{\text {uncorr }}\right)$ is treated as a free parameter. A grid was computed that covered plausible values for $f_{\text {uncorr }}$ and for the maximum entropy regularization parameter. From this grid, images were selected with the best final reduced $\chi^{2}$ value. Using this procedure, the maximum entropy regularizer was not found to significantly improve the image quality or $\chi^{2}$ and thus the submitted images did not explicitly use any regularization (except that intrinsic to the MACIM process).

R Car: The images show a photosphere with a diameter of $\sim 10$ mas. The stellar surface exhibits a patchy structure with significant dark spots located in the South-eastern direction. Around the star, several diffuse concentric rings were detected that might resemble ejecta from past mass-lost events. These shells appear in all wavelength channels at a similar radius from the star, which indicates that at least the two strongest rings are real and not uv-coverage artifacts. Besides the flux from these shell-like circumstellar features no additional uncorrelated flux was included (i.e. $f_{\text {uncorr }}=0$ ).

VY CMa: In the images of VY CMa the stellar surface ( 11 mas) is less well defined than in the images of $\mathrm{R}$ Car, which is likely due to the worse $u v$-coverage and the stronger contributions from extended emission. The stellar surface is patchy, with bright regions in the Northern and Southern hemisphere and a dark spot in the Western direction. At separations of $\sim 32$ mas from the star, we see two blobs that are located along position angle $\sim 25^{\circ}$. These features might belong to the outflow structures that are seen on larger scales (e.g., Ref. 6). However, further checks should be performed to rule out that one of the blobs might be a ghost introduced by insufficient phase information. A slightly better fit was obtained by including an uncorrelated flux component of $f_{\text {uncorr }}=12 \%$. The aperture masking data was not used in the reconstruction process, as it is challenging to include the spatial filtering effect that is introduced by the PIONIER fiber and an ad-hoc treatment might easily introduce artifacts. Further algorithmic work should be undertaken in order to enable a proper reconstruction of such multi-scale data sets, where different spatial filtering constraints need to be associated with different data subsets during the image reconstruction process.

\subsection{Brian Kloppenborg et al. using SQUEEZE-poly $\lambda$}

Brian Kloppenborg and Fabien Baron submitted entries using their software package SQUEEZE ${ }^{26}$ recently modified to allow for wavelength regularization.

For R Car, the object appeared to be a single star. The behavior of the $\mathrm{V}^{2}$ within the second visibility lobe is quite complicated with small-width spikes suggesting well organized small scale structures such as spots. In fact, closure phases show clear departure from symmetry even at short triplet lengths. Adjacent spectral channels do not always follow parallel/adjacent paths through phase-space, thus this object may have some spectrally dependent features. The data was first fitted using SIMTOI with a limb-darkened disk to obtain an initial image. Using this initial image, the SPARCO approach ${ }^{22}$ was tried, i.e. simultaneously fitting a polychromatic stellar disc while its environment is reconstructed. This definitely gave decent images with good reduced $\chi^{2}$. However, because other teams will have done this too, and to encourage diversity, these investigators chose to submit completely model-independent reconstructions. SQUEEZE in polychromatic mode was used, splitting the data into three spectral channels. The SQUEEZE reconstruction used both the uniform disc regularizer ${ }^{28}$ and the transpectral L2 regularizer. ${ }^{21}$ Studying the reduced $\chi^{2}$ during our reconstruction attempts suggested the triple-amplitude errors may be poorly calibrated, and we rescaled them by a factor of 2-3. These investigators report that they wanted to avoid "over-doing it" as they wanted to show what a typical model-independent reconstruction would look like out of SQUEEZE - it is clearly possible to do better. The features believed to be real are: the existence of spots on the surface of the star and some circumstellar emission at larger scales. 
However these investigators did not believe that their submitted images reflected the real flux distribution. More prior information (i.e. in depth-modeling) would be required before more reliable images can be obtained.

For VY CMa, the aperture masking data was not used as it did not appear to be complementary: first, there is a large jump of $\delta V^{2}=0.2$ between 5-6 $\mathrm{M} \lambda$; then, images of the region show that this system is actively losing material, with photometry showing long-term variability trends, and the chances of evolution between the 2008 CONICA and 2014 PIONIER data is highly possible. The PIONIER data does not clearly follow a UDD/LDD pattern as the visibility nulls are not clearly defined, suggesting the target may be intrinsically elongated or asymmetric or that it may present very strong spots. Inspection of $\mathrm{V}^{2}$ plot suggests there could be wavelength dependent features. A LDD model-fit using SIMTOI showed several minima, one around 14 mas and one around 22 mas. Re-fit using two stars in SIMTOI, showed a binary model may even be appropriate for these data, which was found to be somewhat amusing considering the astronomical history of this target, initially thought to be a binary (see e.g. the discussion in Ref. 8) but more recently ${ }^{10}$ shown to be a single stellar disc with extended emission. For this reason, here again a model-independent tri-channel reconstruction was adopted, with total variation as a regularizer. The reconstruction shows one strong source and one weaker source, which was identified as the main star and along with some sort of extended emission (directional jet ? old faint companion ?) - there is a considerable amount of circumstellar material. Overall, the imaging process is limited here by incomplete knowledge of the target. With assurances that the target consisted solely of a stellar disc plus dust shell, here again the SPARCO approach would prove superior.

Note that both the model-fitting code SIMTOI (https://github.com/bkloppenborg/simtoi), and the image reconstruction code SQUEEZE (https://gitorious.org/squeeze/squeeze) are freely available to the community under the GPLv3 licence.

\section{DISCUSSION}

\subsection{General Comments}

From inspection of Figure 5 and Figure 6, we can see many similarities between the entries from the different groups (more on this in the next section). That said, there were also puzzling differences between the entries as well. In the past interferometry beauty contests, ${ }^{25,29-32}$ the "truth" image was known and one could compare the entries to the truth in order to determine which image reconstruction features were artifacts. In practice, the top 2-4 entries were nearly identical, with only fairly subtle differences making the difference between the "winner" and the runner-ups. Here, we see fairly substantial differences between most of the entries. It is clear that "real" data is more challenging and problematic than simulated data, in spite of earlier sophisticated attempts to use realistic error bars, etc.

In this section we attempt to determine what features are most likely to represent actual features of VY CMa and $\mathrm{R}$ Car. The robust participation of 10 independent groups using 4 or 5 different algorithms gives us a unique opportunity to apply the principles of "crowd sourcing" to filter out likely artifacts from real features. Following discussion of each source, we will determine if one of the entries stands out as most compatible with the "group consensus" and crown a tentative "winner" to the 2014 interferometric imaging beauty contest.

\subsection{Construction of "Median" Images}

To aid our analysis, we have constructed median images for each star in each spectral channel. We have done this using the following procedure: a) Re-grid all entries to common 0.1 milliarcsecond pixel scale; b) Align images using cross-correlation optimization (all images convolved with 1.5 mas FWHM Gaussian beforehand only for position alignment - not for median filtering); c) Re-scale images to common stellar surface brightness (this was done because of varying treatments of circumstellar flux in image flux normalization). d) Find median image for the data cube consisting of the 10 entries for each star at each wavelength.

We present the "median images" in Figure 8, along with an overlay of a 11.3 mas diameter circle for reference. ${ }^{10}$ We expect this procedure to yield a high fidelity image within the photosphere of the star where artifacts and noise might reasonable be expected to be equally likely bright or dark compared to the mean. However, for features outside the stellar disk, there is a bias in this method since nearly all algorithms adopted a positive definite constraint in the image reconstructions. Thus, we should interpret structures beyond the disk with more caution in these median images. 


\subsection{VY CMa}

From Figure 5 and Figure 8 we have tentatively identified the following likely "true" features of VY CMa at $\mathrm{H}$ band from the VLTI/PIONIER data:

- Dust shell is over-resolved and no meaningful information was available, except for the fraction of emission in the shell compared to the star.

- The photosphere appears elongated along east-west direction (could be due to optical depth effects from foreground dust, circumstellar molecular emission, temperature variations across photosphere, or something else).

- Two bright spots are on the eastern limb of star.

- While the fraction of light in the shell depends on the wavelength, the features relating to the star itself do not appear strongly chromatic (not quantified here in this paper).

- The $\mathrm{H}$ band diameter is approximately the same size as the near-continuum $\mathrm{K}$ band size reported by VLTI/AMBER ${ }^{10}$ (11.3 mas)

We will refrain from speculating on the interpretation of these images. As discussed earlier, there is a community effort to interpret these data as part of a public ESO process led by Jean-Philippe Berger.

\subsection{R Car}

From Figure 6 and Figure 8 we have tentatively identified the following likely "true" features of R Car at $\mathrm{H}$ band from the VLTI/PIONIER data:

- Prominent circumstellar emission shells. The image consensus seems to be one arc about 12 mas to the East and one about 10 mas to the southwest. Not all contestants are convinced the emission is "arc-like" and more study will need to be done to confirm these geometrical structures and rule out artifacts.

- The shells are significantly stronger in the edge channels of $\mathrm{H}$ band, hardly visible in the center channel.

- The photosphere is mostly circular but with two main spots near the center. The two spots are close together and so could be really one asymmetric, elongated spot.

As far VY CMa, we will refrain from speculating on the interpretation of these images. As discussed earlier, there is a community effort to interpret these data as part of a public ESO process led by Jean-Philippe Berger.

\subsection{A Qualitative Metric based on Consensus}

Using the features identified in this section, JDM and JPB endeavored to compare the contest entries as a way to "score" the beauty contest. If an entry unambiguously reproduced a feature, its as scored a +1 (三smiley face). If an entry partially satisfied the criterion, it scored a +0 (三neutral face). If the entry did not contain the feature, then the score was -1 (三frowning face). The results of this somewhat subjective "scoring*" can be found in Figure 9.

Based on these results, we can announce a winner of the 2014 interferometric imaging beauty contest: Joel Sanchez and his team from IAA-CSIC/Spain using BSMEM. Some combination of the strong use of model-fitting as a first step in creating a prior, the simple gray treatment, or perhaps some final image smoothing imposed by the team seems to have given this team an edge this time. Congratulations!

*JDM/JPB apologize in advance for any mistakes made in constructing this table and proactively beg for mercy from the contestants. 


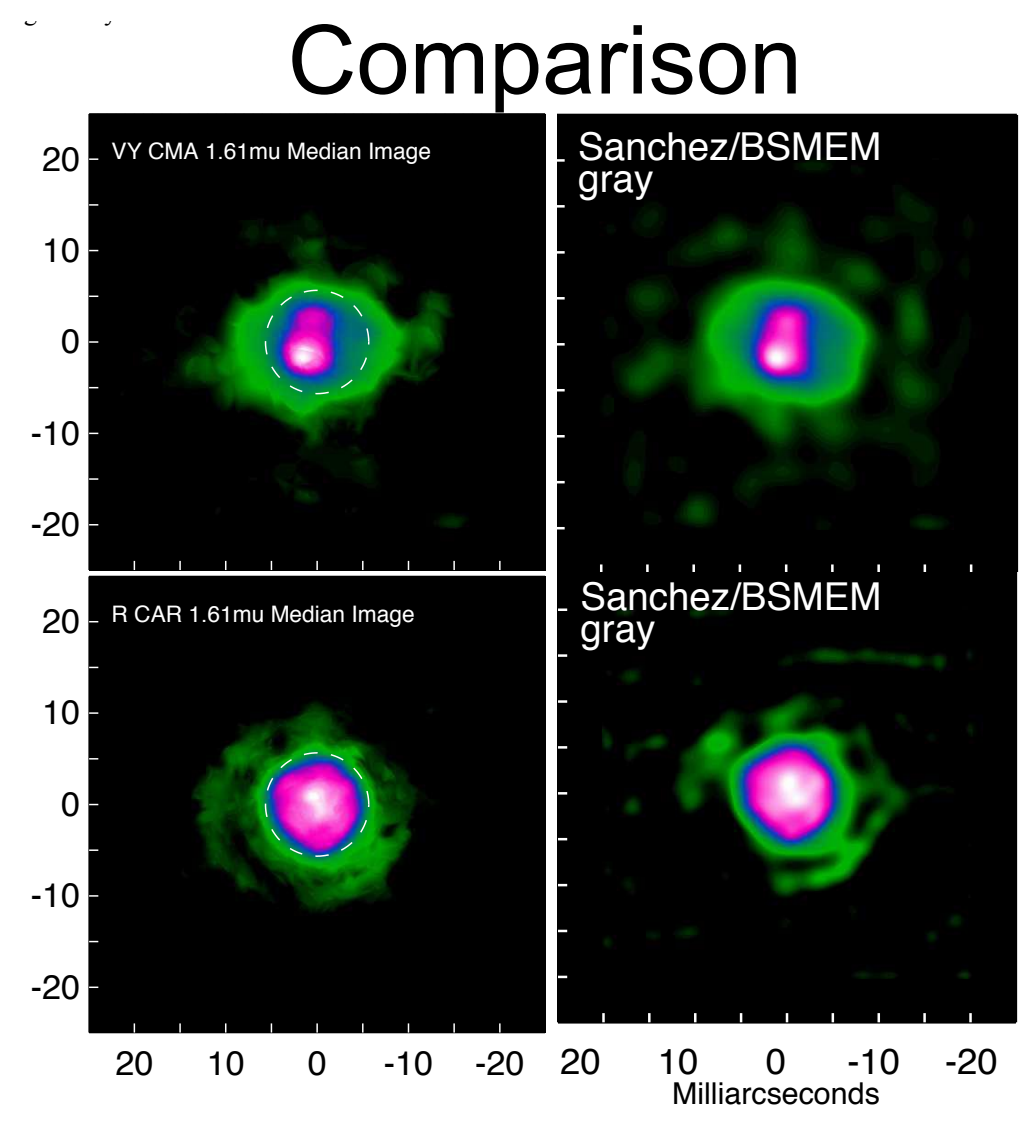

Figure 10. The 2014 Interferometric Imaging Beauty Contest winner is Joel Sanchez-Bermudez and his team from IAACSIC/Spain using BSMEM. Here we compare the winning entry with the "median" images described in the text. Joel and his team reproduced all the main features seen in the median images. We will have to wait for the judgement of history before concluding whether or not these features represent reality or not.

\section{PROSPECTIVE}

We believe that this initial round of image reconstructions for VY CMa and R Car has demonstrated how rich the interpretation of such data could be but also that there is room for improvement. The use of actual data instead of simulated ones has confronted us with the necessity of a second round of reconstructions by the contestants. The next discussion will aim to reach a broad agreement about how to best use the polychromatic data, what priors are best, and a more sophisticated effort to incorporate the aperture masking data for VY CMa. In particular, the shell emission (from water and/or CO) in R Car seems to show similar geometry in the different spectral channels but varies in strength - this kind of information might be better incorporated in the reconstruction process than was done here. It should be noted that a significant fraction of the contestants had limited experience in image reconstruction which is a tribute to the enormous improvement of aperture synthesis techniques for optical interferometry.

Once the contestants have converged to agreed reconstructed images and meaningful features, the images will be handed over to the broader team in order to proceed with the astrophysical interpretation. These initial results are extremely promising in the context of upcoming VLTI instruments GRAVITY and MATISSE. The combination of image reconstruction and spectral resolution offers the perspective of detailed channeled imaging across the molecular/dusty/gaseous layers surrounding stars. We hope that this exercise will contribute to generating trust in the image reconstruction processes and that more astronomers will make use of this capability at VLTI, CHARA and NPOI. 


\section{ACKNOWLEDGMENTS}

We would like to thank Bill Cotton for help at the beginning of the process this year. We also would like to acknowledge ESO's Director for Science and the Paranal Director for releasing the technical time that made this first-of-a-kind imaging experiment possible. Lastly, JDM and JPB would like to thank all the contest entries for going along with this novel experiment and granting the organizers latitude for determining a contest "winner" using admittedly subjective criteria based on dubious metrics!

\section{REFERENCES}

[1] Berger, J.-P., Malbet, F., Baron, F., Chiavassa, A., Duvert, G., Elitzur, M., Freytag, B., Gueth, F., Hönig, S., Hron, J., Jang-Condell, H., Le Bouquin, J.-B., Monin, J.-L., Monnier, J. D., Perrin, G., Plez, B., Ratzka, T., Renard, S., Stefl, S., Thiébaut, E., Tristram, K. R. W., Verhoelst, T., Wolf, S., and Young, J., "Imaging the heart of astrophysical objects with optical long-baseline interferometry," A\&A Rev. 20, 53 (June 2012).

[2] Monnier, J. D., Zhao, M., Pedretti, E., Thureau, N., Ireland, M., Muirhead, P., Berger, J.-P., Millan-Gabet, R., Van Belle, G., ten Brummelaar, T., McAlister, H., Ridgway, S., Turner, N., Sturmann, L., Sturmann, J., and Berger, D., "Imaging the Surface of Altair," Science 317, 342- (July 2007).

[3] Kloppenborg, B., Stencel, R., Monnier, J. D., Schaefer, G., Zhao, M., Baron, F., McAlister, H., ten Brummelaar, T., Che, X., Farrington, C., Pedretti, E., Sallave-Goldfinger, P. J., Sturmann, J., Sturmann, L., Thureau, N., Turner, N., and Carroll, S. M., "Infrared images of the transiting disk in the $\epsilon$ Aurigae system," Nature 464, 870-872 (Apr. 2010).

[4] Le Bouquin, J.-B., Berger, J.-P., Lazareff, B., Zins, G., Haguenauer, P., Jocou, L., Kern, P., Millan-Gabet, R., Traub, W., Absil, O., Augereau, J.-C., Benisty, M., Blind, N., Bonfils, X., Bourget, P., Delboulbe, A., Feautrier, P., Germain, M., Gitton, P., Gillier, D., Kiekebusch, M., Kluska, J., Knudstrup, J., Labeye, P., Lizon, J.-L., Monin, J.-L., Magnard, Y., Malbet, F., Maurel, D., Ménard, F., Micallef, M., Michaud, L., Montagnier, G., Morel, S., Moulin, T., Perraut, K., Popovic, D., Rabou, P., Rochat, S., Rojas, C., Roussel, F., Roux, A., Stadler, E., Stefl, S., Tatulli, E., and Ventura, N., "PIONIER: a 4-telescope visitor instrument at VLTI," A\&A 535, A67 (Nov. 2011).

[5] Pauls, T. A., Young, J. S., Cotton, W. D., and Monnier, J. D., "A Data Exchange Standard for Optical (Visible/IR) Interferometry," PASP 117, 1255-1262 (Nov. 2005).

[6] Monnier, J. D., Tuthill, P. G., Lopez, B., Cruzalebes, P., Danchi, W. C., and Haniff, C. A., "The Last Gasps of VY Canis Majoris: Aperture Synthesis and Adaptive Optics Imagery," ApJ 512, 351-361 (Feb. 1999).

[7] Lacour, S., Tuthill, P., Ireland, M., Amico, P., and Girard, J., "Sparse Aperture Masking on Paranal," The Messenger 146, 18-23 (Dec. 2011).

[8] Smith, N., Humphreys, R. M., Davidson, K., Gehrz, R. D., Schuster, M. T., and Krautter, J., "The Asymmetric Nebula Surrounding the Extreme Red Supergiant Vy Canis Majoris," AJ 121, 1111-1125 (Feb. 2001).

[9] Monnier, J. D., Millan-Gabet, R., Tuthill, P. G., Traub, W. A., Carleton, N. P., Coudé du Foresto, V., Danchi, W. C., Lacasse, M. G., Morel, S., Perrin, G., Porro, I. L., Schloerb, F. P., and Townes, C. H., "High-Resolution Imaging of Dust Shells by Using Keck Aperture Masking and the IOTA Interferometer," ApJ 605, 436-461 (Apr. 2004).

[10] Wittkowski, M., Hauschildt, P. H., Arroyo-Torres, B., and Marcaide, J. M., "Fundamental properties and atmospheric structure of the red supergiant VY Canis Majoris based on VLTI/AMBER spectrointerferometry," A\&A 540, L12 (Apr. 2012).

[11] Ireland, M. J., Tuthill, P. G., Bedding, T. R., Robertson, J. G., and Jacob, A. P., "Multiwavelength diameters of nearby Miras and semiregular variables," MNRAS 350, 365-374 (May 2004).

[12] Le Bouquin, J.-B., Lacour, S., Renard, S., Thiébaut, E., Merand, A., and Verhoelst, T., "Pre-maximum spectro-imaging of the Mira star T Leporis with AMBER/VLTI," A\&A 496, L1-L4 (Mar. 2009).

[13] Perrin, G., Ridgway, S. T., Mennesson, B., Cotton, W. D., Woillez, J., Verhoelst, T., Schuller, P., Coudé du Foresto, V., Traub, W. A., Millan-Gabet, R., and Lacasse, M. G., "Unveiling Mira stars behind the molecules. Confirmation of the molecular layer model with narrow band near-infrared interferometry," A\&A 426, 279-296 (Oct. 2004). 
[14] Hummel, C. A., "A novel imaging algorithm for broadband aperture synthesis data," in [Society of PhotoOptical Instrumentation Engineers (SPIE) Conference Series], Society of Photo-Optical Instrumentation Engineers (SPIE) Conference Series 7734 (July 2010).

[15] Hofmann, K.-H., Weigelt, G., and Schertl, D., "An image reconstruction method (IRBis) for optical/infrared interferometry," A\&A 565, A48 (May 2014).

[16] Wittkowski, M., Langer, N., and Weigelt, G., "Diffraction-limited speckle-masking interferometry of the red supergiant VY CMa," A\&A 340, L39-L42 (Dec. 1998).

[17] Wittkowski, M., Boboltz, D. A., Driebe, T., Le Bouquin, J.-B., Millour, F., Ohnaka, K., and Scholz, M., "J, H, K spectro-interferometry of the Mira variable S Orionis," A\&A 479, L21-L24 (Feb. 2008).

[18] Buscher, D. F., "Direct maximum-entropy image reconstruction from the bispectrum," in [Very High Angular Resolution Imaging], Robertson, J. G. and Tango, W. J., eds., IAU Symposium 158, 91 (1994).

[19] Baron, F. and Young, J. S., "Image reconstruction at Cambridge University," in [Society of Photo-Optical Instrumentation Engineers (SPIE) Conference Series], Society of Photo-Optical Instrumentation Engineers (SPIE) Conference Series $\mathbf{7 0 1 3}$ (July 2008).

[20] Thiébaut, E., "MIRA: an effective imaging algorithm for optical interferometry," in [Society of Photo-Optical Instrumentation Engineers (SPIE) Conference Series], Society of Photo-Optical Instrumentation Engineers (SPIE) Conference Series $\mathbf{7 0 1 3}$ (July 2008).

[21] Thiébaut, É., Soulez, F., and Denis, L., "Exploiting spatial sparsity for multiwavelength imaging in optical interferometry," Journal of the Optical Society of America A 30, 160 (Feb. 2013).

[22] Kluska, J., Malbet, F., Berger, J.-P., Baron, F., Lazareff, B., Le Bouquin, J.-B., Monnier, J. D., Soulez, F., and Thiébaut, E., "SPARCO : a semi-parametric approach for image reconstruction of chromatic objects. Application to young stellar objects," A\&A 564, A80 (Apr. 2014).

[23] Meimon, S., Mugnier, L. M., and Le Besnerais, G., "Convex approximation to the likelihood criterion for aperture synthesis imaging," Journal of the Optical Society of America A 22, 2348-2356 (Nov. 2005).

[24] Meimon, S. C., Mugnier, L. M., and Le Besnerais, G., "Reconstruction method for weak-phase optical interferometry," Optics Letters 30, 1809-1811 (July 2005).

[25] Lawson, P. R., Cotton, W. D., Hummel, C. A., Monnier, J. D., Zhao, M., Young, J. S., Thorsteinsson, H., Meimon, S. C., Mugnier, L. M., Le Besnerais, G., Thiebaut, E. M., and Tuthill, P. G., "An interferometry imaging beauty contest," in [New Frontiers in Stellar Interferometry], Traub, W. A., ed., Society of PhotoOptical Instrumentation Engineers (SPIE) Conference Series 5491, 886 (Oct. 2004).

[26] Baron, F., Monnier, J. D., and Kloppenborg, B., "A novel image reconstruction software for optical/infrared interferometry," in [Society of Photo-Optical Instrumentation Engineers (SPIE) Conference Series], Society of Photo-Optical Instrumentation Engineers (SPIE) Conference Series $\mathbf{7 7 3 4}$ (July 2010).

[27] Ireland, M. J., Monnier, J. D., and Thureau, N., "Monte-Carlo imaging for optical interferometry," in [Society of Photo-Optical Instrumentation Engineers (SPIE) Conference Series], Society of Photo-Optical Instrumentation Engineers (SPIE) Conference Series 6268 (July 2006).

[28] Baron, F., Monnier, J. D., Kiss, L. L., Neilson, H. R., Zhao, M., Anderson, M., Aarnio, A., Pedretti, E., Thureau, N., ten Brummelaar, T. A., Ridgway, S. T., McAlister, H. A., Sturmann, J., Sturmann, L., and Turner, N., "CHARA/MIRC Observations of Two M Supergiants in Perseus OB1: Temperature, Bayesian Modeling, and Compressed Sensing Imaging," ApJ 785, 46 (Apr. 2014).

[29] Lawson, P. R., Cotton, W. D., Hummel, C. A., Baron, F., Young, J. S., Kraus, S., Hofmann, K.-H., Weigelt, G. P., Ireland, M., Monnier, J. D., Thiébaut, E., Rengaswamy, S., and Chesneau, O., "2006 interferometry imaging beauty contest," in [Society of Photo-Optical Instrumentation Engineers (SPIE) Conference Series], Society of Photo-Optical Instrumentation Engineers (SPIE) Conference Series 6268 (July 2006).

[30] Cotton, W., Monnier, J., Baron, F., Hofmann, K.-H., Kraus, S., Weigelt, G., Rengaswamy, S., Thiébaut, E., Lawson, P., Jaffe, W., Hummel, C., Pauls, T., Schmitt, H., Tuthill, P., and Young, J., "2008 imaging beauty contest," in [Society of Photo-Optical Instrumentation Engineers (SPIE) Conference Series], Society of Photo-Optical Instrumentation Engineers (SPIE) Conference Series 7013 (July 2008).

[31] Malbet, F., Cotton, W., Duvert, G., Lawson, P., Chiavassa, A., Young, J., Baron, F., Buscher, D., Rengaswamy, S., Kloppenborg, B., Vannier, M., and Mugnier, L., "The 2010 interferometric imaging beauty contest," in [Society of Photo-Optical Instrumentation Engineers (SPIE) Conference Series], Society of Photo-Optical Instrumentation Engineers (SPIE) Conference Series 7734 (July 2010). 
[32] Baron, F., Cotton, W. D., Lawson, P. R., Ridgway, S. T., Aarnio, A., Monnier, J. D., Hofmann, K.H., Schertl, D., Weigelt, G., Thiébaut, E., Soulez, F., Mary, D., Millour, F., Vannier, M., Young, J., Elias, N. M., Schmitt, H. R., and Rengaswamy, S., "The 2012 interferometric imaging beauty contest," in [Society of Photo-Optical Instrumentation Engineers (SPIE) Conference Series], Society of Photo-Optical Instrumentation Engineers (SPIE) Conference Series 8445 (July 2012). 